\title{
La prevalencia y difusión de la tuberculosis en el Chaco y la ciudad de Resistencia
}

\section{Prevalence and diffusion of tuberculosis in Chaco and Resistencia city}

\author{
Fernando A. Dell'Orto Foschiatti \\ Facultad de Medicina - UNNE \\ Dra. Lic. Liliana Ramírez \\ Prof. Adjunta-Departamento de Geografía \\ Facultad de Humanidades - UNNE \\ Email: Iramirez@hum.unne.edu.ar
}

\section{RESUMEN}

El presente aporte intenta mostrar una aproximación a la situación de la Tuberculosis en la Provincia y en el Área Metropolitana del Gran Resistencia, a partir de datos proporcionados por la Dirección de Estadísticas Sanitarias de la Provincia del Chaco para los años 2000 y 2007. Un pequeño recorrido por la situación a nivel mundial, de los distintos continentes y de la República Argentina nos permite visualizar las diferencias con el contexto provincial y nuestra semejanza con países asiáticos. La Tuberculosis es una enfermedad que encuentra un ambiente propicio para su desarrollo en poblaciones con falta de agua potable, insalubridad de la vivienda y, en especial, con hacinamiento, factores que siguen actuando como agentes propicios para el desarrollo de esta enfermedad.

PALABRAS CLAVE: Tuberculosis - Incidencia - Prevalencia - Mortalidad - Chaco - AMGR.

\section{SUMMARY}

This contribution attempts to show an approach to the situation of Tuberculosis in the Chaco Province and the Great Resistencia Metropolitan Area (GRMA), from data provided by the Directory of Health Statistics of Chaco Province for the years 2000 and 2007. A little tour over the global situation of the different continents and in Argentina we can visualize the differences within the provincial context and our similarity with Asian countries. Tuberculosis is a disease that is environmentally conducive to its development in populations with drinking water shortages, poor housing health and, especially with overcrowding. These factors continue to act, enabling agents to develop this disease.

KEY WORDS: Tuberculosis - Incidence - Prevalence - Mortality - Chaco - GRMA. 


\section{INTRODUCCIÓN}

Desde los inicios de la Ciencia Geográfica se destacó la relación existente con la Medicina, no obstante el origen de esta conexión se remonta al Siglo V .a.C, cuando Hipócrates en su Corpus Hipocrático recurrió a la Geografía para comprender el proceso de las enfermedades y uno de los capítulos más importantes en esta obra es el llamado "Sobre los aires, las aguas y los lugares". Este es el primer documento histórico fundado en la observación y considerado como un primigenio enfoque ambientalista que perdura hasta nuestros días. Con el transcurrir del tiempo la citada relación se fue haciendo cada vez más evidente y la teoría sobre los complejos patógenos, del geógrafo Maximilian Sorre, puede ser calificado como el inicio de la Geografía Médica. Por aquel entonces se daba mayor importancia al estudio de la distribución de las enfermedades y su relación con el ambiente, hasta que en 1949 se cambia la denominación por Geografía de la Salud como una forma de mostrar la importancia que cobro el estudio integral y sistémico de la salud de la población.

En la moderna Geografía de la Salud, aggiornada no sólo con nuevos temas, sino también con temas recuperados y tratados con Tecnologías de la Información Geográfica se pueden distinguir las siguientes especialidades: cartografía médica, ecología médica, epidemiología del paisaje, estudios de difusión, estudios de distribución de los servicios, estudios de simulación y modelado y estudios de utilización. El aporte que presentamos se relaciona con los estudios de cartografía médica y estudios de difusión referida a la Tuberculosis en la Provincia del Chaco.

La tuberculosis (en adelante TB) es una enfermedad que acompañó al hombre durante su desarrollo sobre la tierra. Hay testigos de su presencia en piezas momificadas tanto en Egipto como en Perú. Se afirma que en el mundo cada año mueren más de 2.900.000 personas por TB y que se registran entre 4 y 5 millones de nuevos casos, con una prevalencia que se acerca a los 10 millones. El problema no es igual en todas las regiones: es poco importante en los países desarrollados y muy serio en los subdesarrollados (Gorodner, 1998). Por otro lado la TB es una enfermedad íntimamente ligada a la pobreza. Las condiciones de vida -tanto materiales, como culturales- aparecen como factores determinantes ante la posibilidad de contagiarse esta enfermedad. Son variables de riesgo, por ejemplo, la precariedad de la vivienda, la infraestructura deteriorada, el hacinamiento familiar, la falta de higiene, la ausencia de una cultura de prevención y los problemas nutricionales; además el déficit inmunitario tiene alta incidencia en las chances de contraer la enfermedad, una mala alimentación (poco proteica, desbalanceada, con baja ingesta) se vuelve un factor crucial. (Tierney, 2006) y actúa como un factor que favorece la adquisición de la enfermedad.

En esta contribución se analizará el estado de la TB en los años 2000 y 2007, a partir de la información de morbilidad y mortalidad, por áreas sanitarias y por equipamientos hospitalarios, respectivamente, proporcionada por la Dirección de Estadísticas Sanitarias del Ministerio de Salud de la Provincia del Chaco. 


\section{Objetivos}

1. Precisar la distribución espacial de la TB que caracteriza a la población del Chaco en los años 2000 y-2007.

2. Definir las áreas de posible difusión o inversión de la difusión de la TB en la provincia.

3. Apreciar la evolución de los casos de TB en el Área Metropolitana del Gran Resistencia.

\section{La Tuberculosis ¿Qué tipo de enfermedad es?}

Para realizar investigaciones en Geografía de la Salud es imprescindible conocer el funcionamiento epidemiológico de las enfermedades. Cada una de ellas tiene su propio comportamiento, sus procesos y su tiempo de incubación, en el caso de las infecciosas. La Organización Mundial de la Salud (en adelante OMS) agrupa a la totalidad de los posibles estados morbosos en veintidós capítulos. La TB pertenece a las enfermedades el capítulo I, códigos A00-A99, "ciertas enfermedades infecciosas y parasitarias" y específicamente le corresponde los códigos que van desde el A15 al A19. No obstante existen múltiples tipologías, la morbilidad se clasifica por su origen, por las partes del organismo a las que afecta o por la obligatoriedad, o no, a ser declaradas. Asimismo, es frecuente encontrarlas clasificadas por los términos globalizadores del subdesarrollo, como por ejemplo enfermedades de la urbanización o de la civilización y también conjuntos de enfermedades que hacen referencia a sus características temporales: agudas, crónicas, de larga duración, cíclicas o lentas. También, otra manera más general de dividir las enfermedades, es según su comportamiento:

a) Degenerativas: son las no infecciosas, las de tipo congénito (cáncer, enfermedades cardiológicas).

b) Infecciosas: también llamadas transmisibles o contagiosas. Por su manifestación en el espacio pueden clasificarse también como fenómenos en masa, según se produzca la concentración de casos en el territorio y en función del tiempo.

- Epidémicas: Corresponden a los fenómenos limitados en el espacio y el tiempo. Epidemia es la acumulación de nuevos casos de enfermedad con causa común, por encima de la frecuencia habitual en un lugar dado y en un cierto período.

- Endémicas: Cuando son afecciones que existen habitualmente en una población. Son limitadas en el tiempo y limitadas en el espacio.

- Pandémicas: Se denomina pandemia a los fenómenos de morbilidad que son limitados en el tiempo, pero no el espacio. Su 
área de impacto es el mundo, si bien con enormes diferencias de incidencia ${ }^{1}$.

- Esporádicas: Si sólo se observa de forma dispersa, sin que pueda verse un nexo con casos que tengan causa común.

- Erradicadas: Si ya han sido eliminadas de un territorio. No se producen en ese espacio.

De acuerdo con lo señalado, la TB, que es nuestro objeto de estudio, según su comportamiento es una enfermedad infecciosa y según su característica temporal es endémica.

\section{Historia de la Tuberculosis}

Para analizar la historia de la TB seguiremos a varios autores (http://www.umdnj.edu/ ntbcweb/history.htm). En pocas enfermedades es posible documentar su estrecha relación con la Historia de la propia humanidad como en la que nos ocupa. Existen evidencias paleológicas de TB vertebral en restos neolíticos precolombinos, así como en momias egipcias que datan aproximadamente del año 2400 a.C.

Quizá la primera "cita bibliográfica" que podemos hallar en relación a ella se encuentre en los libros del Antiguo Testamento, donde se hace referencia a la enfermedad consuntiva que afectó al pueblo judío durante su estancia en Egipto, tradicional zona de gran prevalencia de enfermedad.

En Europa se convirtió en un problema grave en el momento en que el hacinamiento en los medios urbanos asociado con la Revolución Industrial generó circunstancias epidemiológicas que favorecieron su propagación. En los siglos XVII y XVIII la TB fue responsable de una cuarta parte de todas las muertes en adultos que se produjeron en el continente europeo (la palabra TB ha sido uno de los grandes "tabúes" en la historia de la cultura occidental).

El médico inglés Benjamín Martenl, en su obra "A New Theory of The Comsumption" fue el primero en aventurar que la causa de la TB podría ser una "diminuta criatura viviente", que, una vez en el organismo, podría generar los signos y síntomas de la enfermedad.

Fue Robert Koch, en 1882, al utilizar una nueva técnica de tinción, el primero que pudo ver al bacilo. En el año 1895 Wilhelm Konrad von Rontgen descubre la radiación que lleva su nombre, con lo que la evolución de la enfermedad podía ser observada.

\footnotetext{
1 "La medición del impacto de las diferentes enfermedades, en el tiempo, espacio e intensidad se realiza mediante indicadores, como el de prevalencia, incidencia y letalidad o mortalidad y tasas de mortalidad específicas y estandarizadas para evitar la distorsión de las diferencias estructurales de la población". (Ana Olivera, 1993:24). La prevalencia, es la proporción de personas que, en un área geográfica, y en un período de tiempo establecido, sufren una determinada enfermedad, se calcula dividiendo el número de individuos que padecen el estado mórbido por la cantidad de habitantes del área considerada. La incidencia mide el número de casos nuevos de una enfermedad en un área geográfica determinada y en un tiempo establecido.
} 
Con el conocimiento del agente causante y el mecanismo de transmisión proliferó la aparición de los famosos sanatorios, con los que se buscaba, por un lado, aislar a los enfermos de la población general interrumpiendo la cadena de transmisión de la enfermedad, y por otro, ayudar al proceso de curación con la buena alimentación y el reposo. Pero no fue hasta 1944, en plena II Guerra Mundial, con la demostración de la eficacia de la estreptomicina, cuando comienza la era moderna de la TB, en la que el curso de la enfermedad podía ser cambiado. En el año 1952 tiene lugar el desarrollo de un agente mucho más eficaz: la isoniacida. Ello hace que la TB se convierta en una enfermedad curable en la mayoría de los casos.

La Rifampicina, en la década de los 60 , hizo que los regímenes terapéuticos se acortaran de una forma significativa. Se produjo un descenso progresivo de casos hasta mediados de los 80 , en los que la irrupción del sida, la inmigración desde países en los que la enfermedad es muy prevalente (no hay que olvidar que la TB es un problema global de la humanidad, de difícil solución con medidas de "fronteras adentro"), la formación de bolsas de pobreza y situaciones de hacinamiento, el impacto en los adictos a drogas por vía parenteral, junto con la escasez de recursos sanitarios, han hecho de la TB un problema creciente, con la adquisición y propagación epidémica de nuevos casos.

\section{Características epidemiológicas de la enfermedad}

La TB es causada por bacterias pertenecientes al complejo de Mycobacterium Tuberculosis infectando a un número estimado de 20 a $43 \%$ de la población mundial. Al año, más de 2,9 millones de personas mueren por la enfermedad en todo el mundo. Se presenta en forma desproporcionada entre personas en poblaciones con desventajas, como desnutridos, desvalidos, indigentes, marginados, y otros individuos que habitan aglomerados en hogares con condiciones deplorables. Existe una producción cada vez mayor de TB entre individuos infectados con HIV / SIDA (Síndrome de Inmunodeficiencia Adquirida).

Esta enfermedad suele asentar en los pulmones, pero en el $33 \%$ de los casos afecta a otros órganos. La infección con Mycobacterium Tuberculosis se inicia cuando una persona susceptible inhala gotitas dispersas en el aire cuyos núcleos contienen organismos viables. Los bacilos de la TB que alcanzan los alvéolos son fagocitados por macrófagos alveolares, y se produce infección cuando las bacterias inoculadas escapan de la actividad microbicida de estos macrófagos. Una vez que se establece la infección, se produce en forma típica, su diseminación linfática y hematógena, antes de que se desarrolle una respuesta inmunitaria eficaz. En esta etapa de la infección, llamada TB primaria, suele ser clínica y radiográficamente silenciosa (Tierney, 2006: 249).

Los individuos con esta infección de TB latente no tienen una enfermedad activa y no pueden transmitir los organismos a otras personas. Sin embargo, es posible que la infección se reactive si se produce deterioro en las defensas inmunitarias del huésped. Cerca del $10 \%$ de los individuos con TB latente que no recibe terapia preventiva, desarrollará TB activa durante el transcurso de su vida y en la mitad de los casos esto sucede dentro de los dos años siguientes a la infección primaria. 
Hasta un $50 \%$ de los pacientes infectados con el virus del HIV desarrollará TB activa en un plazo de dos años posterior a la infección con el bacilo de la TB. Diversos trastornos, como la gastrectomía, silicosis y diabetes mellitus y estados acompañados de depresión inmunitaria se relacionan con aumento en el riesgo de reactivación. En cerca del $5 \%$ de los casos, la respuesta inmunitaria es inadecuada y el huésped desarrolla TB primaria progresiva, acompañada por síntomas pulmonares y constitucionales. Además aumentó el porcentaje de pacientes con presentaciones atípicas, en particular ancianos, pacientes con infección por HIV y aquellos que habitan en asilos (Tierney, 2006: 250).

\section{Situación de la Tuberculosis a nivel mundial y regional}

La TB se constituye en un auténtico problema de salud pública, tanto a nivel local, nacional como mundial, por lo que es necesario analizar su situación epidemiológica actual que es muy importante para comprender correctamente esta enfermedad. Este análisis se realiza examinando los principales indicadores que son la tasa de prevalencia, incidencia y mortalidad o letalidad (1).

Según los datos de la OMS, en el período 2000-2007, la prevalencia de esta enfermedad es la que se puede apreciar en el gráfico 1. Como podemos observar el promedio mundial, inferior a 200 por cien mil habitantes, con tendencia descendente, es el resultado del comportamiento de la enfermedad en todos los continentes. El continente africano presentaba una tendencia ascendente hasta el 2003, descendió a una tasa de 400 y se mantuvo en este valor hasta el 2007. En el otro extremo, la región europea presentaba tasas que, al iniciar el período analizado, alcanzaban el valor de 53 por cien mil habitantes y su marcha descendente culminó con un valor de 37 en 2007.

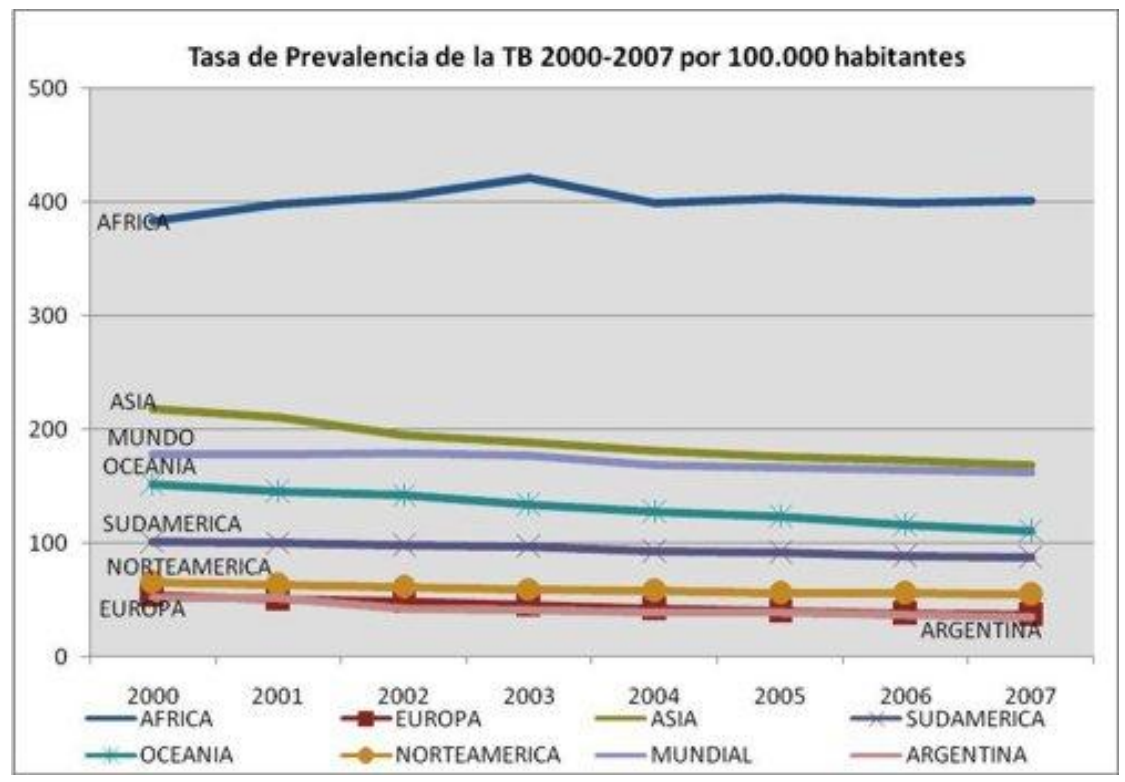

Gráfico 1. Fuente: OMS. www.who.int/es/ 
En cuanto a la incidencia, según estimaciones de la OMS, la Región de Asia Sudoriental registró el mayor número de nuevos casos de TB, correspondiéndole el $34 \%$ de la incidencia mundial. Sin embargo, la tasa de incidencia estimada en el África subsahariana es casi el doble que en la Región de Asia Sudoriental, con cerca de 350 casos por 100000 habitantes. En el año 2001 se informó a la OMS de más de 3.8 millones de casos de TB que incluyeron todas las formas (pulmonar y extrapulmonar). El $90 \%$ de ese total provenían de países en desarrollo. Sin embargo, ante el bajo nivel de detección y los informes incompletos, los casos registrados representan solo una fracción del total. Se ha calculado que en el 2005 se produjeron en el mundo 8.8 millones de casos nuevos de la enfermedad, 95\% de los cuales surgieron en países en desarrollo de Asia (4,9 millones), África (2,6 millones), Oriente Medio (0.6 millones) y América Latina (0.4 millones). También se ha estimado que en el año 2005 se produjeron 1.6 millones de fallecimientos por TB, $95 \%$ de los cuales se produjeron en países en desarrollo.

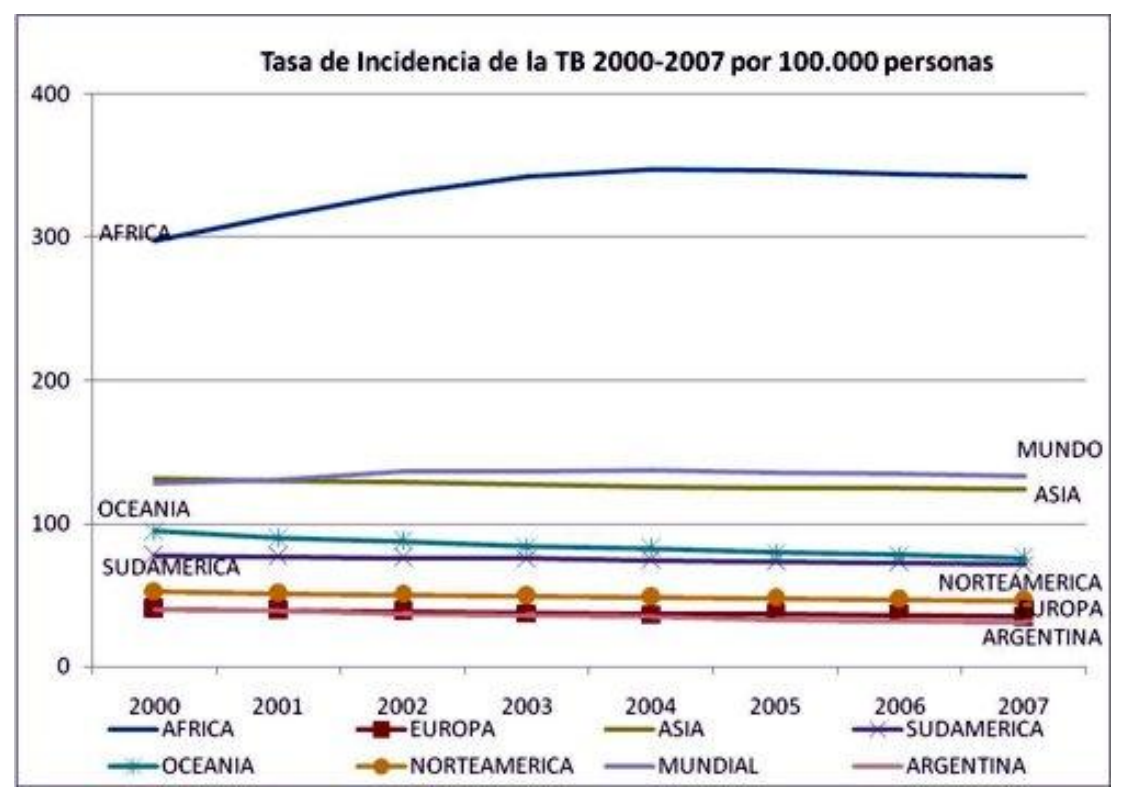

Gráfico 2. Fuente: OMS. www.who.int/es/

En 2005, la incidencia estimada de TB por habitante era estable o decreciente en las seis regiones de la OMS. No obstante, esa lenta disminución está contrarrestada por el crecimiento de la población. Como consecuencia, sigue aumentando el número de nuevos casos por año a escala mundial y en las regiones de la OMS de África, el Mediterráneo Oriental y Asia Sudoriental.

La TB es poco frecuente entre los jóvenes adultos de origen europeo, que sólo rara vez han estado expuestos durante los últimos decenios, a la infección por el Mycobacterium Tuberculosis. Sin embargo, esta enfermedad ha reaparecido en Europa, causando un importante problema en salud pública, debido ante todo a los casos registrados en inmigrantes procedentes de países con una gran prevalencia de la enfermedad. En cambio, debido al alto riesgo que existió tiempo atrás, la prevalencia de la infección tuberculosa es bastante alta entre los ancianos de origen caucásico, quienes siguen estando más expuestos a padecer una TB activa. 
Las tendencias recientes de la enfermedad en países emergentes denotan estabilidad, casi sin disminución de la frecuencia, con dos excepciones. En primer lugar, en los países africanos del sur del Sahara, la epidemia cada vez más amplia de infección por HIV ha hecho que se duplique y aun se triplique el número de casos de TB registrados en los últimos 15 años. En segundo lugar, en países de la antigua Unión Soviética y en Rumania el número de casos ha mostrado un aumento al doble o triple en los últimos 10 años, principalmente a causa del deterioro de la situación socioeconómica y de la infraestructura asistencial (Harrison, 2007).

En cuanto a la mortalidad o letalidad de la enfermedad se calcula que 1,6 millones de personas murieron por TB en 2005, siendo la región africana la que registró el mayor número de muertes y de mortalidad. La epidemia de TB en África avanzó rápidamente en los años 1990, pero el crecimiento se ha frenado poco a poco cada año, y ahora las tasas de incidencia parecen haberse estabilizado o haber comenzado a descender. En el gráfico siguiente podemos apreciar que el continente africano presenta la mayor tasa de mortalidad entre 79 y 87 defunciones por cien mil habitantes en el período considerado con tendencia ascendente, esta región es seguida por Asia, Oceanía, Sudamérica, Norteamérica y Europa, en esta última región las tasas pasaron de 6 a 5 defunciones por cien habitantes con marcha descendente.

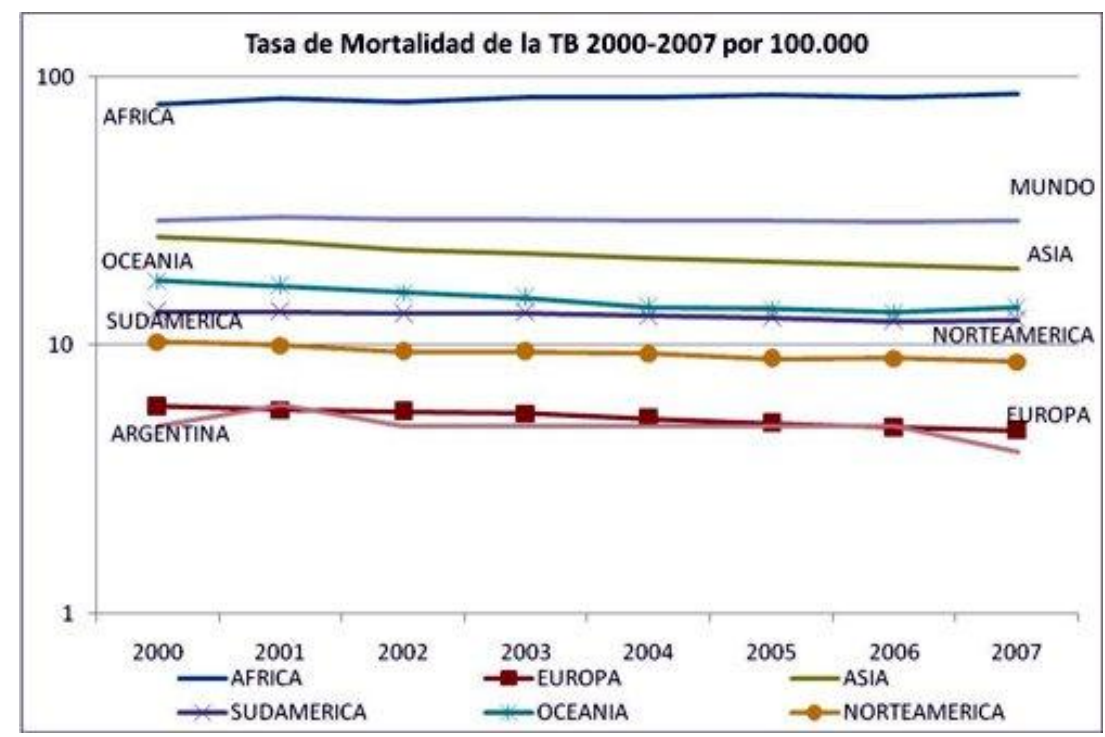

Gráfico 3. Fuente: OMS. www.who.int/es/

La OMS y sus socios internacionales han creado el Grupo de Trabajo sobre TB/VIH con el fin de desarrollar una política mundial de control de la TB relacionada con la infección por el VIH cuyo principio se asienta en la lucha contra ambas infecciones para combatir su combinación letal. La política interna sobre actividades de colaboración TB/VIH describe pautas para crear mecanismos de colaboración entre los programas de TB y $\mathrm{VIH}$ y reducir las cargas de TB en la población y de infección por VIH en pacientes tuberculosos. 


\section{Situación de la Tuberculosis en la República Argentina}

En nuestro país, la TB es un importante problema de salud pública por el daño que provoca, tanto por su morbilidad (número de personas enfermas, que a su vez transmiten la enfermedad), como por su mortalidad (personas que mueren como consecuencia directa o indirecta por las secuelas que esta deja). El daño que provoca esta enfermedad y sus consecuencias mortales, son producidas por una afección que tiene un diagnóstico sencillo y un tratamiento eficaz en la gran mayoría de los casos. En salud pública se la considera como el mejor ejemplo de enfermedad social, por estar relacionada a la pobreza, malnutrición, factores culturales y falta de atención primaria como así también, falta de difusión y prevención. Según la OMS, el $90 \%$ de los enfermos de TB, podría curarse con el tratamiento oportuno, pero sólo el $27 \%$ recibe medicación.

Para analizar los casos de prevalencia, incidencia y mortalidad en los años considerados veamos la tabla y el gráfico siguiente, donde se representa la tasa por cien mil habitantes para cada caso considerado.

\begin{tabular}{|c|c|c|c|c|c|c|c|c|}
\hline & $\mathbf{2 0 0 0}$ & $\mathbf{2 0 0 1}$ & $\mathbf{2 0 0 2}$ & $\mathbf{2 0 0 3}$ & $\mathbf{2 0 0 4}$ & $\mathbf{2 0 0 5}$ & $\mathbf{2 0 0 6}$ & $\mathbf{2 0 0 7}$ \\
\hline PREVALENCIA & 52 & 51 & 42 & 41 & 39 & 39 & 37 & 35 \\
\hline INCIDENCIA & 40 & 39 & 37 & 36 & 35 & 33 & 32 & 31 \\
\hline LETALIDAD & 5 & 6 & 5 & 5 & 5 & 5 & 5 & 4 \\
\hline
\end{tabular}

Tabla 1: Tasa de prevalencia, incidencia y mortalidad de la TB. Fuente: OMS. www.who.int/es/

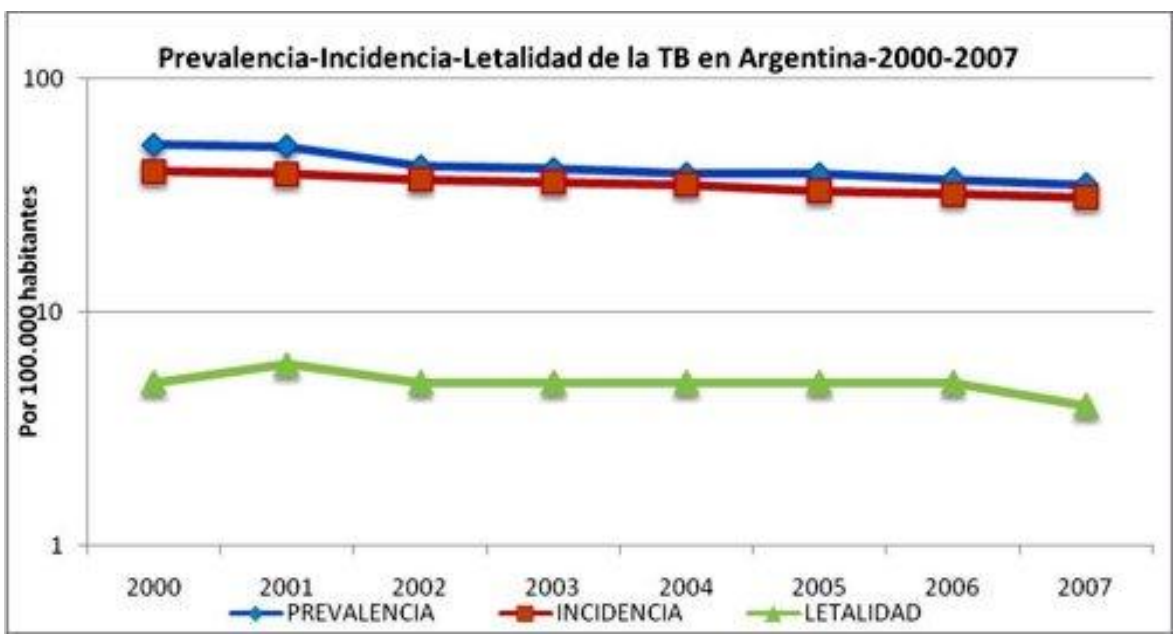

Gráfico 4 (escala logarítmica). Fuente: OMS. www.who.int/es/

Advertimos que tanto la prevalencia como la incidencia presentan una marcha descendente, en el primer caso de 52, en el año 2000, se pasó a 37 por cien mil en el año 2007, mientras que en el segundo caso de 40 se pasó a 31 por cien mil en los mismos momentos. Si observamos la tasa de mortalidad la misma presenta una 
mayor estabilidad entre 5 y 4 por cien mil habitantes al inicio y al final del período respectivamente, con un pequeño ascenso en el año 2001.

Con el fin de comparar la situación argentina con alguna de las regiones que analizamos en el punto anterior, hemos incluido en los gráficos anteriores 1, 2 y 3, la curva que muestra la evolución de la situación nacional, allí se puede apreciar que tanto en la prevalencia, como en la incidencia y en la mortalidad la Argentina es comparable con la situación europea, no obstante, esta media nacional esconde los disímiles escenarios provinciales, como veremos seguidamente.

\section{Situación de la Tuberculosis en el Chaco}

\section{Morbilidad}

En 2000 se produjeron un total de 443 egresos por TB, mientras que en el año 2007, fueron 356. En ambos momentos analizados más del 65\% de los egresos correspondió a los equipamientos localizados en los Departamentos San Fernando, Comandante Fernández y General Güemes. En el gráfico inferior vemos que el Hospital "4 de Junio-Dr. Ramón Carrillo" de Presidencia Roque Sáenz Peña es el que atiende un mayor número de enfermos, 141 en el año 2000 y 95 en el año 2007, seguido por el Hospital "General Güemes" de Juan José Castelli, que tuvo un total de 119 pacientes en el año 2000 y 69 en el año 2007. A diferencia de estos dos nosocomios, cuyos egresos disminuyeron, se advierte que en el caso de los hospitales localizados en la capital provincial los egresos han aumentado en ambos años considerados. Finalmente, en el total de los restantes hospitales y puestos sanitarios A que cubren la Provincia el número también disminuyó de 134 en el año 2000 a 119 en el año 2007.

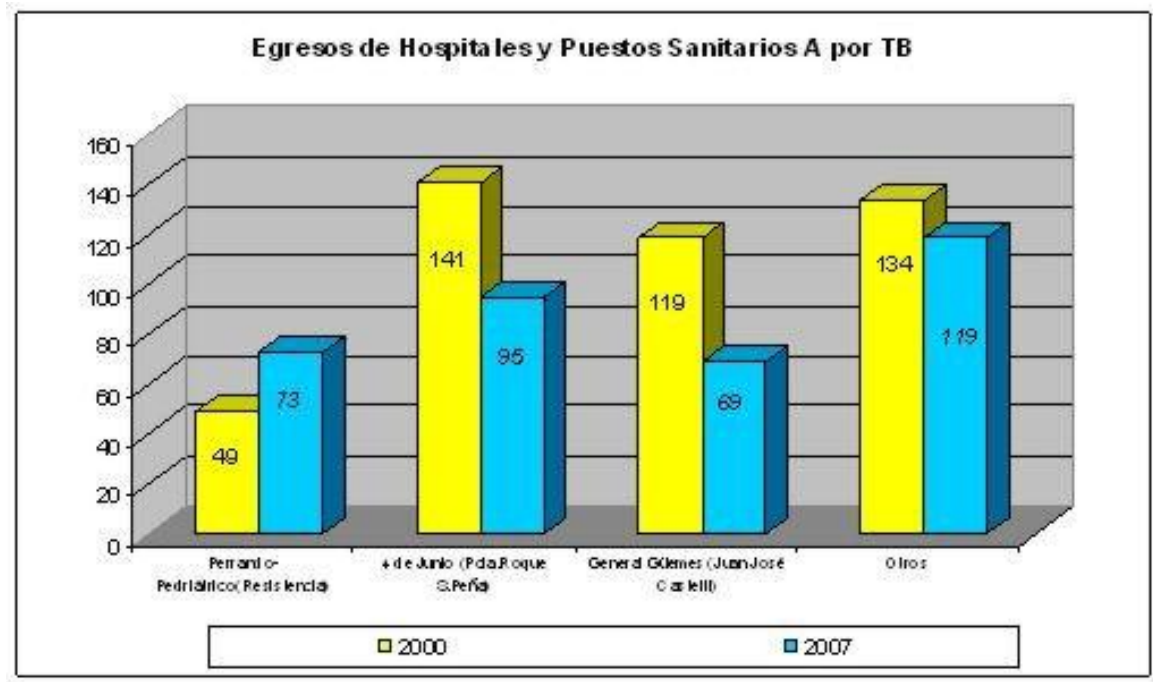


Estos casos en números absolutos se traducen en una altísima tasa de morbilidad ya que si relacionamos los casos citados con la población susceptible de padecer la enfermedad logramos la distribución espacial de la enfermedad en la provincia (2000 y 2007) con una alta incidencia en el área oeste y centro del territorio.

Las representaciones expuestas más abajo reflejan la distribución espacial y la evolución temporal de la enfermedad en la provincia. Como se expresara, los departamentos con mayores tasas son los del norte y oeste, quizá asociados con la mayor cantidad de población indígena excluidos y aislados en áreas de extrema pobreza. El período de transición entre 2000 y 2007 denota una importante disminución en el registro de casos en los departamentos del sudoeste (Mapas 1 y 2). Si consideramos el contexto internacional expuesto en el inicio de este aporte, podemos señalar que la situación provincial se asemeja a la del continente asiático.

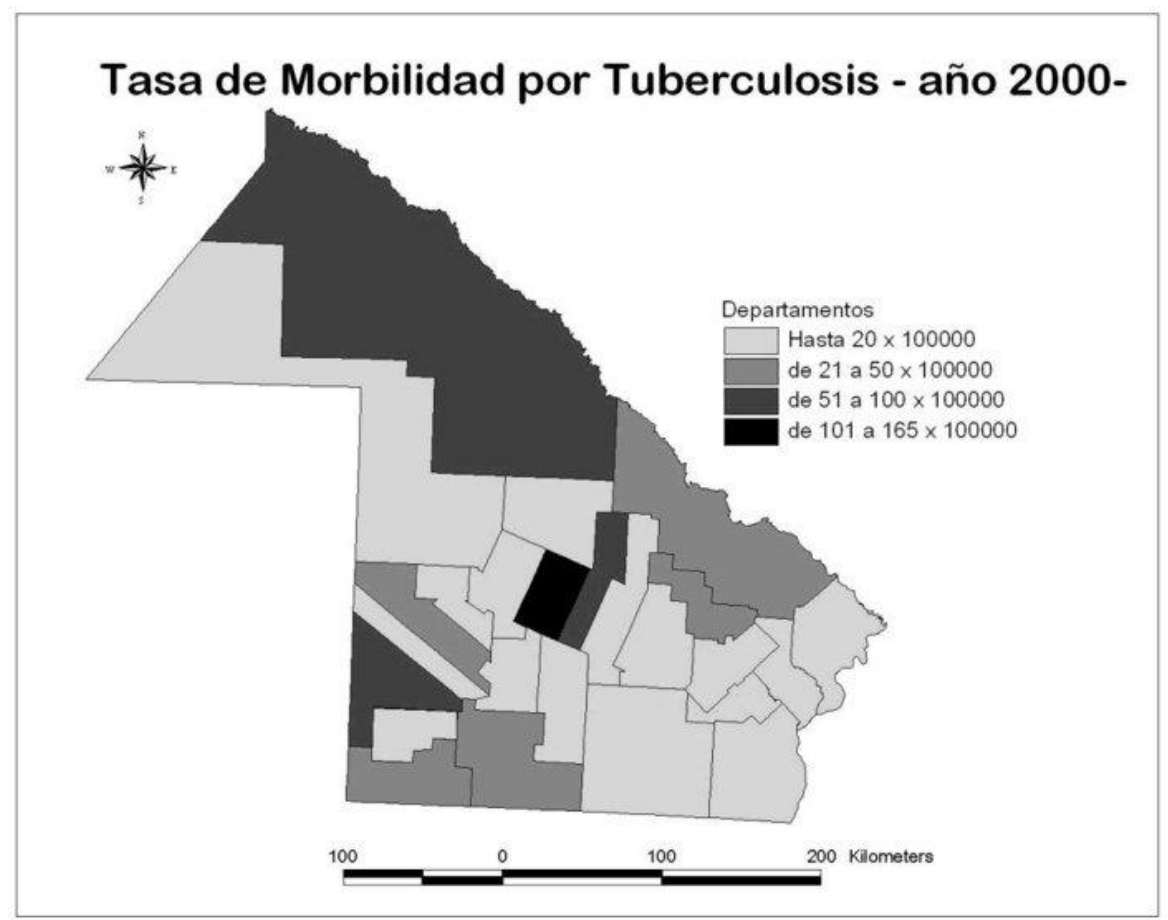

Mapa 1. Fuente: Dirección de Estadística Sanitaria de la Provincia del Chaco, 2000 


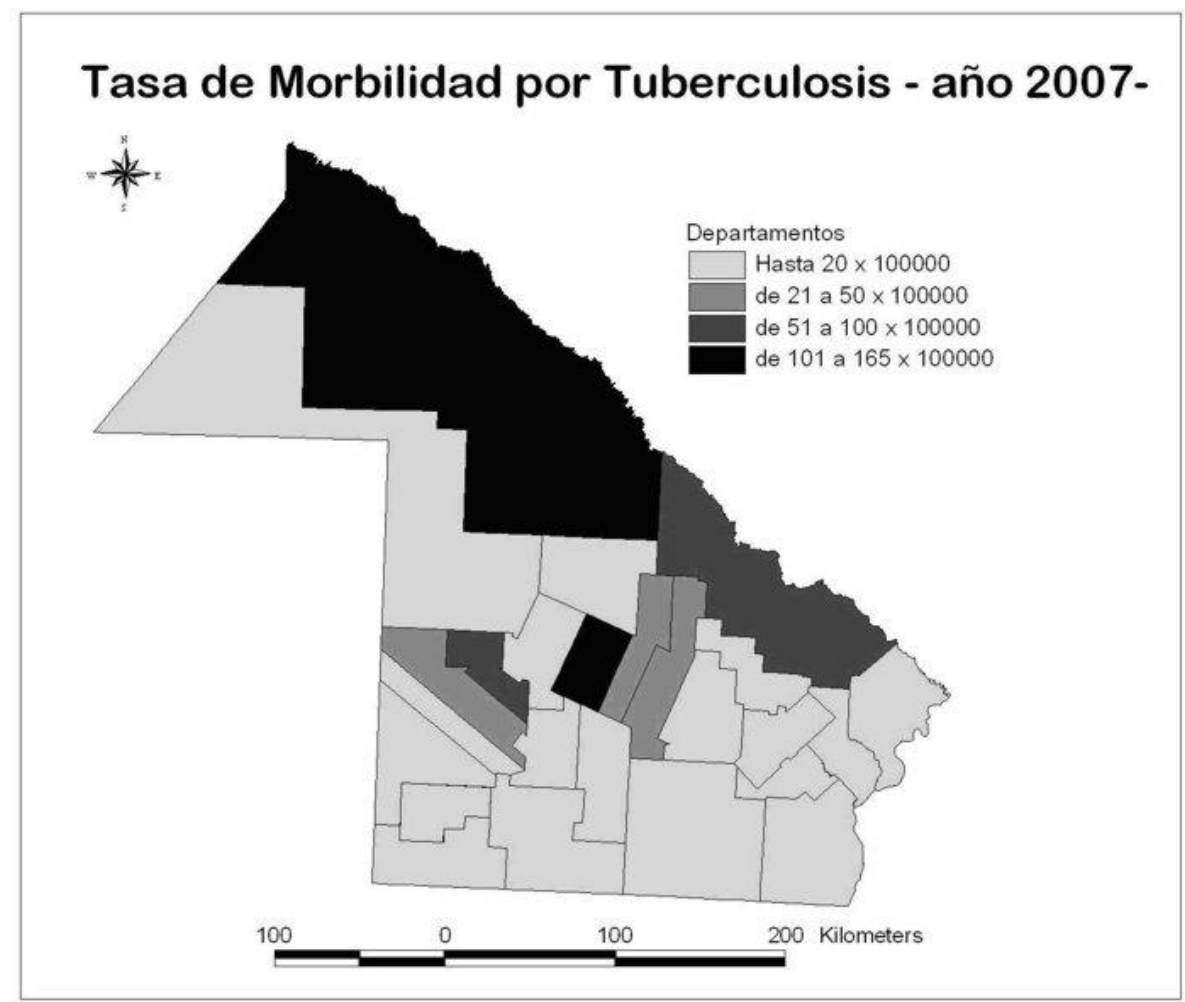

Mapa 2. Fuente: Dirección de Estadística Sanitaria de la Provincia del Chaco, 2007

Este escenario condujo a que el Gobierno del Chaco y del Ministerio de Salud de la Nación solicitaran la intervención a la Organización Panamericana para la Salud (OPS), Oficina Regional de la OMS; tras ella, se elaboró un informe que fue producido por la misión y presentado a comienzos de abril de 2008 ante las autoridades nacionales y provinciales. En él se destaca que en la actualidad, la TB se encuentra "fuera de control"; que no se efectúa profilaxis familiar, identificación de casos, ni búsqueda activa de sintomáticos respiratorios. En el informe recordaron que en 1965 una gran epidemia de TB enfermó a 500 aborígenes, esta circunstancia mereció la implementación de un programa desarrollado por la iglesia protestante conducido por el doctor Humberto Cichetti, quién, con su equipo, realizó un aporte trascendente, este hecho sigue siendo la única experiencia de política sanitaria registrada para las comunidades aborígenes.

\section{Mortalidad}

Actualmente la OPS señala que el gobierno de la Provincia había presentado un grave cuadro de situación, sobresaliendo los efectos mortales de la TB y el Mal de Chagas, que son dos endemias fuertemente enraizadas en la región. A estas patologías, el informe agrega otras enfermedades como la desnutrición, la anemia y la hipertensión, derivados de la falta o de la insuficiencia de alimentos, de la monodieta hidrocarbonada, de las fallas estructurales del sistema sanitario y de la atención brindada a los sectores vulnerables (http://www.who.int/es/). La OPS llegó a 
la conclusión de que el sistema sanitario chaqueño no cuenta con el número de profesionales suficiente para atender a las poblaciones de El Impenetrable. También advirtieron que el Estado todavía continúa en la etapa de reunir la información de las distintas áreas, lo que ha generado la preocupante situación de que existe crisis de decisión, que se traduce en respuestas o acciones excesivamente lentas frente a la gravedad de la emergencia. También hallaron niveles de enfrentamientos y de fragmentaciones en el Estado, hasta con internas políticas, lo que en definitiva ha dificultado la formulación de acciones públicas ante un escenario tan complejo como es el que rodea a las comunidades indígenas.

En el relevamiento de la OPS, se corroboró que la pobreza extrema y el hambre crónico son atendidos por medio de un sistema de políticas públicas profundamente deshumanizadas, que produjeron consecuencias letales en las comunidades indígenas, que, combinado con otros factores, aumentó la mortalidad por enfermedades que son propias del subdesarrollo, mencionándose a la TB, el mal de chagas, la desnutrición, a la que se agrega el SIDA -con una alta incidencia-, en aumento, y que se relaciona directamente con la TB.

El equipo técnico de la OPS señaló que se potencia este cuadro generalizado por la falta de una adecuada infraestructura de servicios para las poblaciones indígenas y excluidos sociales. La misión resaltó que todo el esquema debilita la capacidad de gestión, lo que deriva en una situación altamente conflictiva por la brecha que existe entre las expectativas de los pueblos originarios, las acciones y los discursos que emanan del Ministerio de Salud; esa brecha detectada genera fuertes discordias producto de que no se auspicia la participación de las comunidades, que se reconocen segregadas.

En los mapas 3 y 4 se observa la distribución espacial y la evolución temporal de la mortalidad por la enfermedad que nos ocupa, en los dos momentos estudiados, años 2000 y 2007. Es clara la disminución en algunos sectores, aunque surgen algunos departamentos con un leve aumento en la incidencia de muertes por TB. EI área occidental y central son las más afectadas en ambas fechas.

Así considerando los tipos de difusión de las enfermedades en el espacio (expansión, re-localización o contracción), podemos señalar que lo que más caracteriza a esta enfermedad en el territorio chaqueño es la relocalización. No se advierte una clara contracción y tampoco podemos hablar de expansión ya que en términos de valores absolutos y relativos se ha advertido una disminución. 


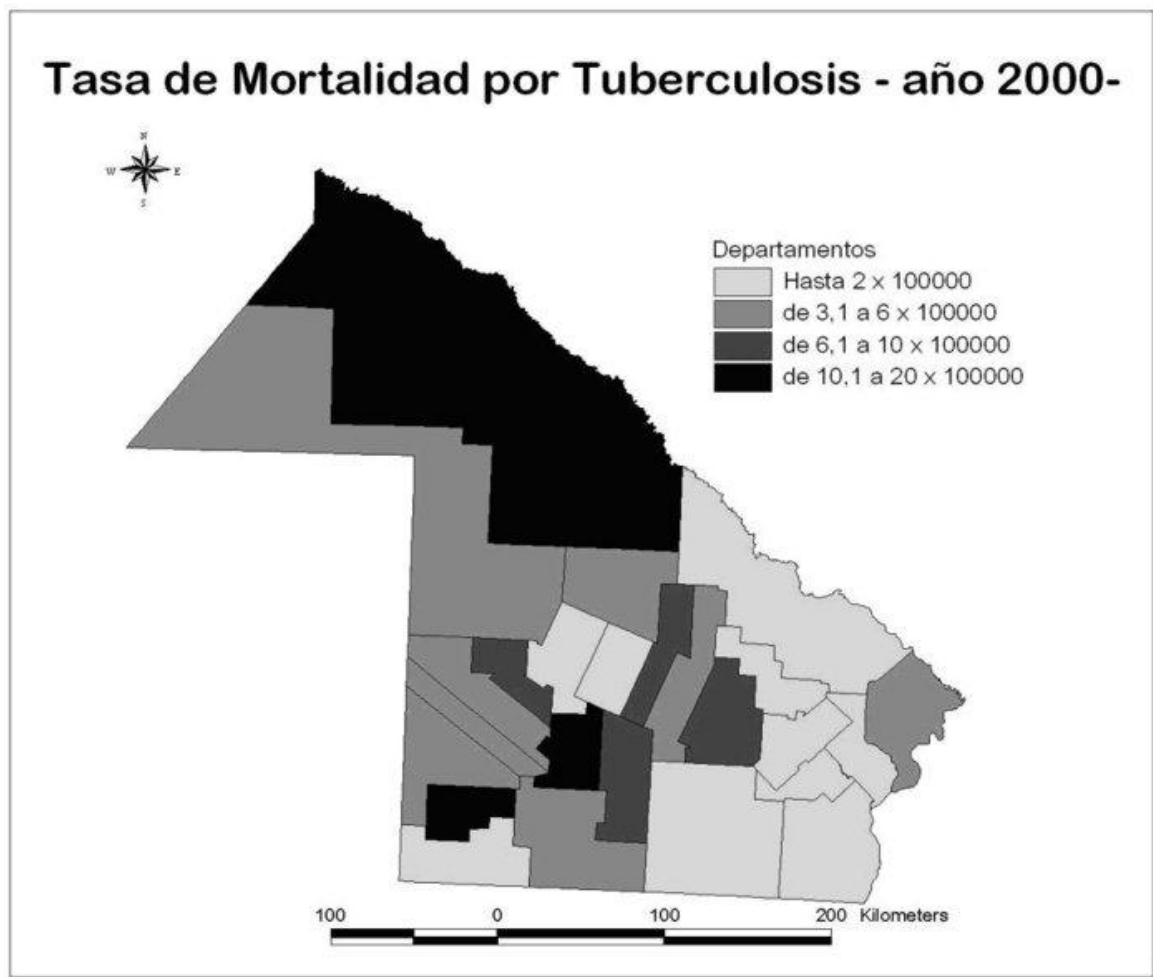

Mapa 3. Fuente: Dirección de Estadística Sanitaria de la Provincia del Chaco, 2000

Tasa de Mortalidad por Tuberculosis - año 2007-

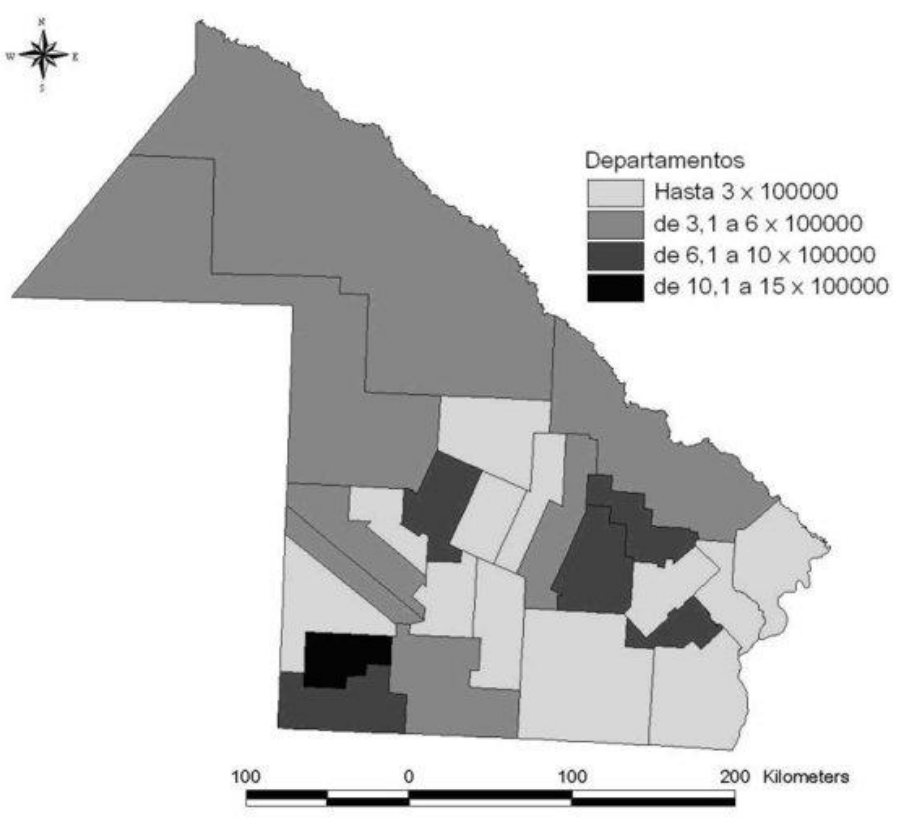

Mapa 4. Fuente: Dirección de Estadística Sanitaria de la Provincia del Chaco, 2000 


\section{Situación de la Tuberculosis en el Área Metropolitana del Gran Resistencia}

\section{Morbilidad}

La TB sigue el patrón epidemiológico del iceberg, según el cual, podemos conocer el número de fallecidos por la enfermedad, con más dificultad también el número de enfermos, pero sería casi imposible precisar el número de sujetos infectados por el Mycobacterium Tuberculosis. Los egresos por TB en los hospitales públicos de Resistencia aumentaron considerablemente su número entre el año 2000 y el 2007. La mayor proporción está representada por los enfermos notificados en el Hospital Perrando, que atiende a población residente en la capital, aunque un buen número de pacientes provienen del interior provincial. En el hospital Pediátrico, que atiende niños hasta los 12 años, no se observa gran variación, aunque sí un leve descenso.

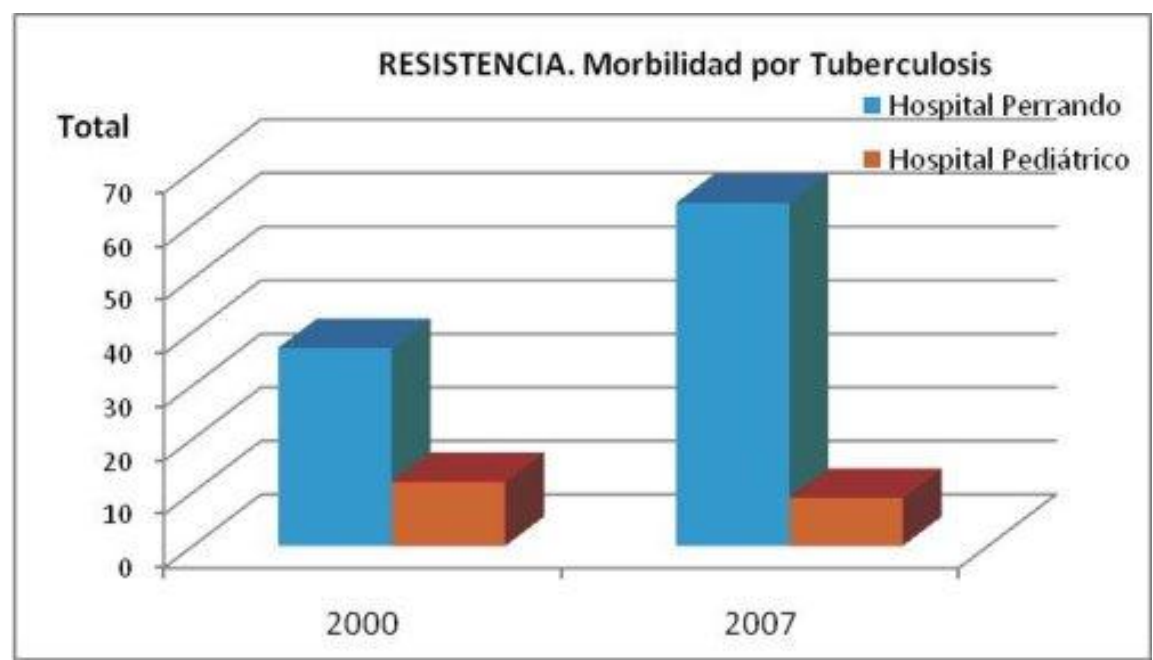

Gráfico 6: Fuente: Dirección de Estadística Sanitaria de la Provincia del Chaco, 2000-2007

La TB del niño tiene características peculiares, porque aunque es de poca contagiosidad y desde el punto de vista de salud pública puede ser de escaso interés, es un excelente indicador de cómo se encuentra la TB en una comunidad y del éxito o fracaso de las medidas de control adoptadas. Ante un niño infectado o enfermo de TB debe realizarse un estudio de sus contactos para identificar su caso índice, que por lo general será un adulto. Los niños cobran especial importancia, ya que detrás de todo niño suele haber un adulto bacilífero. La TB infantil se presenta como una enfermedad difícil de identificar, ya que la infección suele ser asintomática y permanece latente, y la sintomatología clínica y los signos radiológicos no son específicos de la enfermedad. Esta suele ser por lo general una consecuencia directa de la TB del adulto, principalmente del enfermo bacilífero, por lo que a mayor número de fuentes de contagio, mayor será la proporción de niños que se encuentren en riesgo de contagio, infección y enfermedad. En el gráfico anterior se puede observar un mínimo descenso del índice de morbilidad pediátrica por TB que revela una incoherencia respecto de índices de morbilidad más 
elevados en pacientes adultos. Esto supone un riesgo potencial de los niños a contraer la enfermedad, por lo que la estrategia de control de la enfermedad debe ser lo más amplia posible. El riesgo o posibilidad de desarrollar la enfermedad en sujetos infectados por el Mycobacterium Tuberculosis varía en función de la edad, siendo muy elevado en los dos primeros años de la vida. Niños mayores, entre 3 y 4 años, y en la pubertad, aunque igual de sensibles que los menores, tienen menor probabilidad de desarrollar la enfermedad. En adolescentes y adultos jóvenes el riesgo de que la infección evolucione hacia la enfermedad es nuevamente elevado. Teóricamente se pueden prevenir todos los casos de TB, para ello bastaría con controlar la enfermedad en los adultos.

\section{Mortalidad}

La mortalidad ocasionada por TB de los enfermos atendidos en los hospitales capitalinos aumentó significativamente, tal lo observado en el gráfico siguiente.

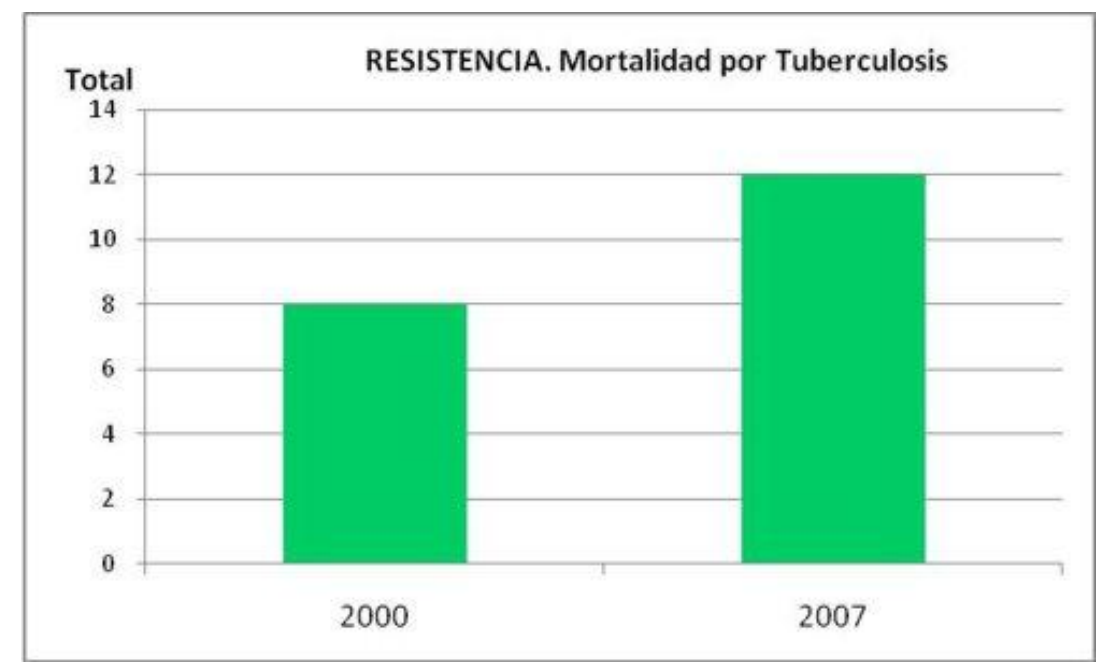

Gráfico 7. Fuente: Dirección de Estadística Sanitaria de la Provincia del Chaco, 2000-2007

Asimismo, la incidencia de esta enfermedad en los diferentes grupos etareos se hace sentir en las edades adultas, principalmente en los mayores de 50 años, tanto en los valores observados en Chaco como en Resistencia (Gráfico 8) 


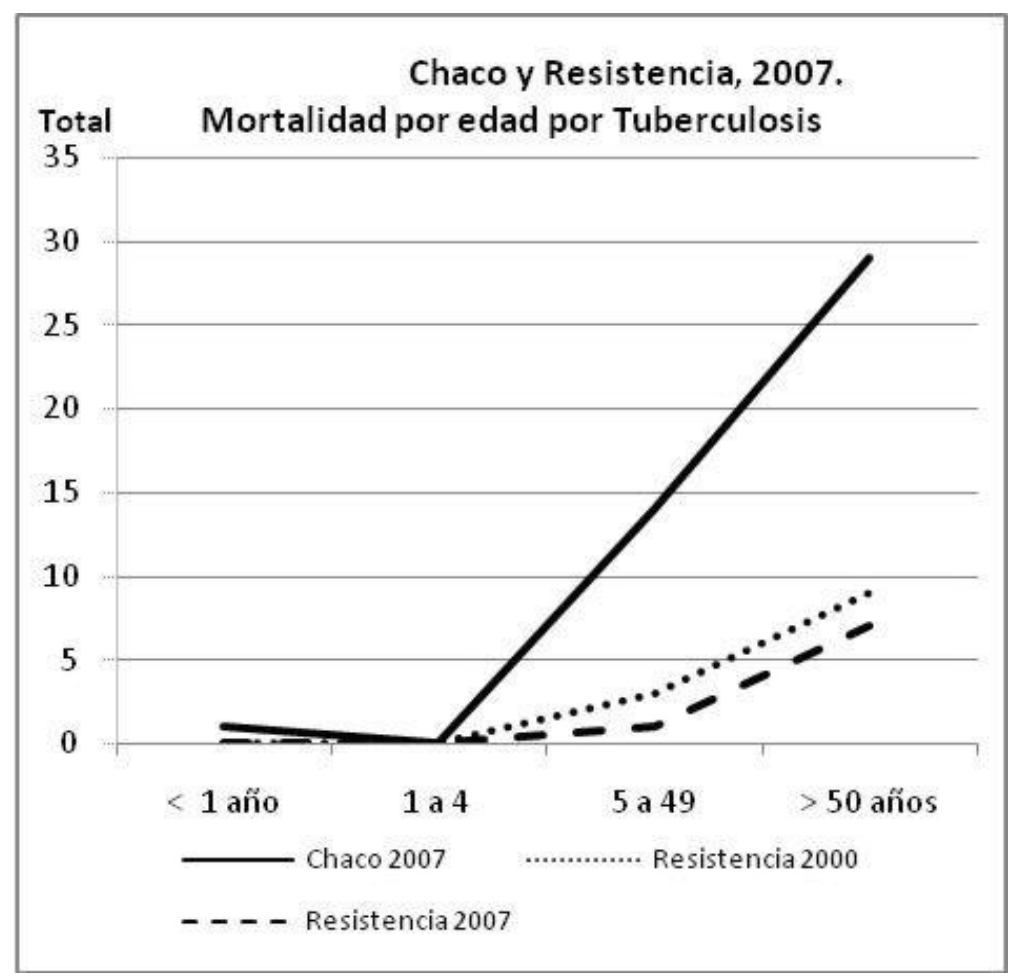

Gráfico 8. Fuente: Dirección de Estadística Sanitaria de la Provincia del Chaco, 2007

Seguramente los más afectados son los grupos más vulnerables perjudicados por los efectos de la pobreza y todas las consecuencias que ella provoca en la salud de la población, no solo por la falta de alimentación apropiada, las prácticas de higiene y saneamiento inadecuadas, sino que además la falta de cobertura médica y las necesidades básicas insatisfechas, deterioran temprana y progresivamente la salud de la población.

\section{CONCLUSIONES}

Los resultados de este trabajo nos permiten sostener que la connotación social y ambiental de estas afecciones es substancial. La consideración de los elementos del ambiente y los factores socioculturales afectan a las sociedades en el espacio y en el tiempo. La TB es una enfermedad que encuentra un ambiente propicio para su desarrollo en poblaciones con falta de agua potable, insalubridad de la vivienda $\mathrm{y}$, sobre todo, con hacinamiento, factores que actuaron en la provincia como elementos mórbidos de las poblaciones marginadas.

Los grupos más empobrecidos de la población chaqueña habitan en estructuras edilicias precarias y con espacios reducidos, por lo que poseen los índices más altos de hacinamiento, por lo general la alimentación es deficiente, a lo que suma la baja cobertura de servicios básicos que reciben y las enfermedades asociadas a la contaminación de las aguas y/o acumulación de basuras; todas estas características están íntimamente ligadas a los niveles más altos de mortalidad. 
La educación sanitaria es uno de los mecanismos de prevención cuya difusión debe ser potenciada. La campaña de educación sanitaria dirigida a la población general debe enfatizar que la TB continúa siendo un problema de salud importante, que su conocimiento va a contribuir a que los sujetos con síntomas de la enfermedad y aquellos que presentan un riesgo aumentado acudan a los servicios de salud. Debe remarcarse que la atención médica, el diagnóstico y el tratamiento son gratuitos, y que la enfermedad se cura completamente cuando el tratamiento se realiza correctamente.

Sin embargo, la puesta en práctica de este tipo de asistencia es difícil. La falta de recursos necesarios para implementar verdaderas políticas de salud, dejó a la acción sanitaria provincial resumida al mantenimiento de los equipamientos sanitarios generalmente descuidados, limitándose a una acción de tipo caritativa y fragmentaria, con tratamientos curativos antes que preventivos. Por ello la TB persiste en aquellas áreas con menores recursos, empobrecidas y carentes de la atención mínima necesaria para tratar la enfermedad. Tales son los departamentos del oeste provincial, donde la mayoría de la población se encuentra privada de las condiciones básicas de salubridad que impiden el desarrollo de esta enfermedad y ello determina, como pudimos observar en la cartografía, las altas tasas de morbilidad y mortalidad comparables con países asiáticos subdesarrollados. Ante esta situación la propagación y persistencia de esta patología se difunde cada vez más, ya que encuentra los factores adecuados para su expansión y relocalización.

\section{BIBLIOGRAFÍA}

ABRAHAM, Rosa; ACOSTA, Liliana Y PICKENHAYN, Jorge A. [1999] Aplicación de un modelo de difusión espacial a patologías con incidencia ambiental y social en la Provincia de San Juan, en "Contribuciones científicas", Gæa, Sociedad Argentina de Estudios Geográficos, San Juan.

ACOSTA, Liliana [2000] La tuberculosis desde la perspectiva de la geografía médica", en "Contribuciones científicas, Gæa, Sociedad Argentina de Estudios Geográficos, Mar del Plata, pp. 13 a 24.

ALAIS, Ma.Eugenia; RHODIUS, Edgardo; PRIETO, Ernesto. Epidemiología de la Tuberculosis. Servicio de Neumonología del Hospital Alemán.

GORODNER, Jorge Osvaldo (Dir.) (1998). Enfermedades Infecciosas, Vol I, EUDENE-Graffit S.R.L., Buenos Aires.

HAGGET, Meter (1988). Geografía. Una síntesis moderna. Capítulo 13. Difusión espacial. Hacia una convergencia regional. Editorial Omega. Barcelona, España. Pp. 311-333.

HARRISON, Tinsley R. (2006) Principios de Medicina Interna, Vol. I, 16 Edición, McGraw-Hill Interamericana, Editores S.A. México. 
HARRISON, Tinsley R. (2007). Principles of Internal Medicine, 17th edition. http://accessmedicine.com

INE. Instituto Nacional de enfermedades respiratorias "Dr Emilio Coni". Ministerio de Salud de la Nación. La Tuberculosis en la República Argentina. Mayo 2006.

IÑIGUEZ ROJAS, Luisa y BARCELLOS, Christovam (2003), Geografía y salud en América Latina: evolución y tendencias. En Revista Cubana de Salud Pública, Cuba, Centro de Estudios de Salud y Bienestar Humanos de la Universidad de La Habana, №29 (4).

NUNES C [2007] Tuberculosis incidence in Portugal: spatiotemporal clustering. En: International Journal of Health Geographic, 6:30, July 2007

OLIVERA, Ana. (1993) Geografía de la Salud. Espacios y Sociedades, Serie general número 26, Editorial Síntesis, Madrid.

TIERNEY, L.; MCPHEE S. Y PAPADAKIS, M. (2006) Diagnóstico clínico y tratamiento. 41ed. Manual Moderno, México.

PEREZ PADILLA, José Rogelio (2003) Altitud y salud. Ejemplo con la mortalidad con tuberculosis y neumonía en México. Boletín de los Sistemas Nacionales Estadístico y de Información Geográfica, Vol.2, №1.

PICKENHAYN, Jorge y CURTO, Susana (2006) La geografía de la salud en Argentina. En Revista Geográfica del Instituto Panamericano de Geografía e Historia, México DF, №138, pp. 89 a 108.

PUTRUele, Ana María (coord.) (2000). Situación de la tuberculosis en la República Argentina. En $8^{\circ}$ Congreso Internacional de medicina interna, 60, Supl.1:27-41, Buenos Aires.

RAMíREZ, Mirta Liliana [2001] La eficacia de los servicios hospitalarios. Un intento por definir las áreas con déficit de recursos en la Provincia del Chaco. En: Revista Científica de la Universidad de Ciencias Empresariales y Sociales -UCES- Vol. V, № 1. Pp 108-135. Buenos Aires.

RAMÍREZ, Mirta Liliana [2006] Geografía de la salud del Chaco. Una aproximación al perfil sanitario y epidemiológico de la población chaqueña y a la utilización de los servicios hospitalarios. Univ. Nac. del Nordeste. Facultad de Humanidades. ISBN 987-05-0451-5. Resistencia, Chaco.

RAMÍREZ, Mirta Liliana [2007] La vulnerabilidad sanitaria y epidemiológica. Aportes metodológicos que permiten definir las áreas sanitarias y epidemiológicas críticas y la población en riesgo en la Provincia del Chaco. Aceptado para publicar. En: 
Aportes conceptuales y empíricos de la vulnerabilidad global. (Foschiatti, Ana María -Autora-Compiladora-). ISBN 978-950-656-108-6, EUDENE Corrientes.

RODRIGUEZ CRUZ, RODOLFO (2004) Enfermedades infecciosas. Tuberculosis. Metas del Milenio. OMS, http://www.paho.org

Salud y Sociedad (2007). Diario La Nación. http://www.bioetica.org/.

TELLES MAS, FERRAZOLI L, WALDMAN EA y colaboradores (2005). A Population-Based Study of Drug Resistance and Transmission of Tuberculosis in an Urban Community. International Journal of Tuberculosis and Lung Disease 9(9):970-976, Sep 2005.

WHO-OMS Organización Mundial de la Salud http://www.who.int/es/

A su vez, se recurrió a distintos sitios web que aportaron una visión más actual del fenómeno estudiado. Se visitó páginas web provinciales, nacionales e internacionales:

Centro de Investigaciones Epidemiológicas de la Academia Nacional de Medicina, Universidad de Buenos Aires. http://www.cie.anm.edu.ar

$\begin{array}{lllll}\text { Organización Panamericana de la Salud (OPS) } & \end{array}$ http://www.paho.org/default_spa.htm

Programa de Geografía Médica, Universidad Nacional de San Juan. http://www.ffha.unsj.edu.ar/GeoMed/inicio.htm

Revista Brasilera de Geografía de la Salud: Hygeia http://www.hygeia.ig.ufu.br

Revista Cubana de Salud Pública http://www.bvs.estudios.geomed.htm

Universidad Nacional de San Juan - Argentina http://www.ffha.unsj.edu.ar/ GeoMed/inicio.htm

National Tuberculosis Center.http://www.umdnj.edu/ ntbcweb/history.htm. 1996 NJMS 